[Vicino Oriente XXI (2017), pp. 127-145]

\title{
TOMBEAUX ET INSCRIPTIONS FUNÉRAIRES DE GHAZNI (AFGHANISTAN). QUELQUES DOCUMENTS INÉDITS DU XI ${ }^{\text {- }}$ XIII ${ }^{\mathrm{e}}$ SIÈCLE
}

\author{
Roberta Giunta - Université de Naples « L’Orientale »
}

This paper deals with twenty-two Islamic tombs which the Italian Archaeological Mission in Afghanistan recorded in Ghazni. These artefacts, despite their frequently fragmentary state, further contribute to our knowledge of medieval Islamic funerary and epigraphic workmanship in the eastern Iranian region.

Keywords: Ghazni; Afghanistan; Ghaznavids; tombs; epitaphs

En 2003 a paru une étude monographique sur les tombeaux découverts par la Mission Archéologique Italienne dans la ville de Ghazni, en Afghanistan (1957-1978), datables entre la fin du $\mathrm{X}^{\mathrm{e}}$ siècle et la première moitié du XIII ${ }^{\mathrm{e}}$ siècle ${ }^{1}$. Ces monuments funéraires en marbre blanc, compact et dur, furent souvent retrouvés en bon état de conservation dans leur emplacement d'origine. Ils ont permis de faire de la lumière sur une tradition architectonique funéraire qui prit naissance à Ghazni dès l'arrivée de la dynastie ghaznévide (977-1186) et qui ne semble pas trouver des comparaisons dans les autres régions islamiques, à l'époque dont il est question ${ }^{2}$.

Dès 2004, grâce à la reprise des activités de la Mission en Afghanistan et à la constitution du projet archéologique Islamic Ghazni de l'IsIAO de Rome ${ }^{3}$, il a été possible d'effectuer un réaménagent de la totalité de la documentation d'archive et d'entreprendre un recensement des matériaux, surtout dans le but d'assurer leur sauvegarde. Ces travaux ont également permis d'acquérir des données nouvelles et de parvenir à une connaissance beaucoup plus approfondie d'un patrimoine dont la valeur historique et artistique est indiscutable. Parmi les « découvertes », nous avons enregistré un petit lot de tombeaux d'époque médiévale, entiers ou fragmentaires, qui font l'objet de cette étude et représentent un élargissement du corpus publié en $2003^{4}$.

La qualité des photographies étant variable, il n’a pas été toujours possible d'avoir une reproduction complète de ces monuments funéraires ; par conséquent, le déchiffrement des épitaphes est parfois partiel.

1 Giunta 2003. Cette monographie était le résultat d'une thèse de doctorat soutenue à l'Université Aix-Marseille en novembre 1999, sous la direction de Solange Ory (Giunta 1999).

2 Giunta 2000.

3 Dès lors, la Mission Italienne est dirigée par Anna Filigenzi ; la direction du projet Islamic Ghazni m’a été confiée par Maria Vittoria Fontana.

4 Dans les dernières années, Martina Massullo a conduit une recherche importante sur 177 tombeaux de la ville datés à la période post-mongole (Massullo 2017). Toutes ces études montrent qu'aucune ville de l'Afghanistan actuel n'a livré autant de tombeaux à épitaphes s'échelonnant entre la fin du $\mathrm{X}^{\mathrm{e}}$ siècle et l'époque moderne, même si la plupart d'entre eux ne demeure plus sur place. 


\section{TOMBEAUX ENTIERS}

Lors d'une prospection menée en 1966, l'une des faces longitudinales de deux tombeaux a été prise en photo (fig. 1, 2) ${ }^{5}$. Dans les deux cas, l'épitaphe était gravée sur l'élément de couronnement. Le premier tombeau (inv. IG485) se composait de trois éléments entourés par des blocs en pierre : un socle à deux degrés dépourvu de décors et d'inscriptions, un socle prismatique orné d'une séquence de motifs végétaux gravés en creux et un couronnement monolithique mouluré. Une plaque rectangulaire, également en marbre et sans doute ajoutée postérieurement, marquait la position de la tête du défunt (fig. 1). Le mauvais état de conservation du bloc de couronnement - cassé en trois morceaux - ne permet qu'une lecture partielle de cette partie du texte, qui est sculptée en relief à l'intérieur d'un bandeau rectangulaire. L'écriture est en caractères cursifs, sans points diacritiques. Des décors végétaux comblent les espaces vides entre les lettres, dans la moitié supérieure du bandeau :

$$
\begin{aligned}
& \text { حسام الدولةو الدين [...] الثرق علي بن [ابر هـ] بيم ؟ رحمه الله } \\
& \text { Husām al-dawla wa'l-dīn [...] al-šarq 'Alī b. [Ibrah]īm ?. Que Dieu ait pitié de lui ! }
\end{aligned}
$$

Cette partie de l'épitaphe contient les laqabs, le ism et le nasab du défunt, suivis d'une bénédiction en sa faveur. Les cassures du bloc rendent difficile le déchiffrement du deuxième laqab et du nasab.

Dans le deuxième tombeau (inv. IG491) plusieurs éléments sont assemblés et il est impossible de savoir s’ils étaient cohérents ${ }^{6}$. La photo reproduit un bloc de couronnement monolithique et mouluré qui reposait au-dessus d'un socle à degrés; des plaques rectangulaires, partiellement enterrées, étaient disposées autour des deux éléments, en guise d'enclos ; ces plaques, qui devaient originairement délimiter le soubassement de la tombe, portent une décoration - qui semble avoir été laissée inachevée - composée de petites édicules évoquant le profil d'une niche de mihrā $b^{7}$. Plusieurs blocs en pierres étaient éparpillés sur le côté nord de la tombe, en correspondance avec la tête du défunt (fig. 2). L’écriture est en caractères cursifs très minces, pourvus de points diacritiques.

$$
\begin{aligned}
& \text { * عالم الغيب و الثهادة* هو الرحمن الرحيم } \\
& \text { *Il est celui qui connaît ce qui est caché et ce qui est apparent* (Cor. XIII, 9) }{ }^{8} \text {. Il est } \\
& \text { le Bienfaiteur, le Miséricordieux. }
\end{aligned}
$$

Le verset coranique - dont le texte n'offre que la partie initiale - ne se rencontre pas dans les autres épitaphes anciennes de la ville ${ }^{9}$. Il en va de même pour l'exaltation de

5 Aucun renseignement ne permet de remonter à la localisation de ces deux monuments qui, dès lors, semblent avoir disparu.

6 En ce qui concerne la question des remplois d'éléments en marbre, voir Giunta 2003, 333-334 et tombeaux n 71-77.

7 Une décoration similaire est témoignée sur les plaques du soubassement de certains tombeaux de la première moitié du XIII ${ }^{\mathrm{e}}$ siècle (Giunta 2003, n 58, 62, 63, 74, C.1-C.13, C.15, C.19-C.21).

$8 \quad$ Ici et infra, pour la traduction des versets coraniques nous avons utilisé D. Masson, Le Coran, Paris 1967.

9 Un tombeau du XII ${ }^{\mathrm{e}}$ siècle retrouvé dans la ziyārat Huwāğa Muhammad Kalbī à Bāì-i Bihišt, dans la zone occidentale de la ville, porte le verset 24 de cette sourate (Giunta 2003, n³2). 
Dieu en tant que bienfaiteur et miséricordieux ${ }^{10}$. Le nom du défunt était probablement gravé sur la face opposée.

Trois tombeaux (inv. IG1811, IG57, IG6200) furent découverts dans des sondages de fouille conduits en 1969 par la Mission, sous la direction de Maurizio Taddei, à proximité de la ziyāra de Šams, au nord-ouest de la citadelle. Les trois monuments, ensevelis à moins d'un mètre de profondeur, se conservaient presque intacts dans leur emplacement d'origine, parfaitement orientés ; il restait également un bon nombre de plaques en marbre constituant le dallage du petit espace funéraire (fig. 3). En 2004 les blocs de couronnement de deux de ces tombeaux (IG1811 et IG57) ont été retrouvés par la Mission dans les dépôts du Musée National de Kaboul. Nous ignorons la localisation des autres éléments qui, en l'état actuel, semblent avoir disparu. Dans tous les cas, l'épitaphe, sculptée en relief dans un même style d'écriture en coufique feuillu, figure uniquement sur les faces longitudinales des couronnements, à l'intérieur de bandeaux rectangulaires ${ }^{11}$.

Inv. IG1811. Tombeau composé de cinq éléments : un soubassement délimité par des plaques verticales, un socle/couvercle, un socle à deux degrés, un socle prismatique et un bloc de couronnement mouluré. Exception faite du socle à deux degrés, les trois autres éléments inférieurs offrent une riche décoration : une séquence d'arcs trilobés, entrelacés en leur sommet et sculptés en relief, orne les plaques du soubassement ; des motifs végétaux gravés en creux suivent le contour des quatre faces supérieures du socle/couvercle, ainsi que les quatre faces du socle prismatique. Un large fleuron polylobé est sculpté en relief sur les deux petits côtés supérieurs de ce dernier. L’épitaphe ne contient que huit mots (fig. 46).

Couronnement, face est :

$$
\begin{gathered}
\text { ملك [...] عبد ملك [...] Abd Malik } \\
\text { Malik }
\end{gathered}
$$

Couronnement, face ouest :

$$
\text { *كل نفس ذائقة الموت }
$$

*Tout homme goûtera la mort* (Cor. XXIX, 57).

La première partie de cette épitaphe, qui pourrait se référer au défunt, pose des problèmes de déchiffrement. Toutes les lettres du deuxième mot ont la forme de segments verticaux dépourvus de points diacritiques; les deux graphies possibles, شناتين ou ne sont pas convaincants. L'expression Tout homme goûtera la mort est empruntée à deux autres versets coraniques (Cor. III, 185 et Cor. XXI, 35) et trouve plusieurs attestations dans les épitaphes de la ville, ce qui laisse imaginer qu'elle devint à l'époque une formule stéréotypée ${ }^{12}$.

Inv. IG57. Tombeau composé de cinq éléments : un soubassement délimité par des plaques verticales, un socle/couvercle, un socle simple, un socle prismatique et un bloc de couronnement mouluré. Les plaques du soubassement portent la même séquence d'arcs

10 Ces deux épithètes divines se retrouvent uniquement dans la basmala.

11 D’après une habitude fréquente, les faces latérales des couronnements de ce type sont souvent dépourvues de décors et d'inscriptions (voir Giunta 2003, 349-352).

12 Voir Giunta 2003, 365. 
trilobés et entrelacés qui figure sur le soubassement du tombeau précédent. Des motifs végétaux gravés en creux suivent le contour des quatre faces supérieures des deux socles, ainsi que les quatre faces du socle prismatique.

Couronnement, face est (fig. 7) :

$$
\begin{gathered}
\text { كل نفس* } \\
\text { *Tout homme }
\end{gathered}
$$

Couronnement, face ouest (fig. 8) :

$$
\text { ذائقة المـوت }
$$

$$
\text { goûtera la mort* (Cor. XXIX, 57). }
$$

Inv. IG6200. Tombeau composé de cinq éléments, dont la forme, les dimensions et la décoration sont identiques à celles du tombeau précédent.

Couronnement, face est (fig. 9) :

$$
\begin{gathered}
\text { محمد بن احمد } \\
\text { Muhammad b. Aḥmad }
\end{gathered}
$$

Couronnement, face ouest (fig. 10) :

$$
\begin{aligned}
& \text { عبد السلام } \\
& \text { 'Abd al-Salām. }
\end{aligned}
$$

Différemment des deux autres tombeaux de cet espace funéraire, l'épitaphe révèle uniquement le nom du propriétaire de la tombe.

Un autre tombeau entier enregistré dans les archives de la Mission Italienne (inv. IG6201) a été pris en photo en 1978 par Francesco Noci dans la ziyārat 'Alī Lālā, pas loin d'autres tombeaux datés à une période postérieure (fig. 11) ${ }^{13}$. De ce monument il ne restait qu'un bloc de couronnement monolithique et mouluré, cassé en deux parties inégales placées au-dessus de deux blocs en marbre dont celui de droite appartenait à un socle prismatique peu élevé. Soit le bloc, soit le morceau de socle portent une inscription en écriture cursive dépourvue de points diacritiques ; néanmoins, les dissemblances entre les deux styles de graphie laissent supposer l'appartenance des deux éléments à deux tombeaux différents.

\section{Couronnement :}

$$
\text { لا اله الا الله محمد رسول الله }
$$

Il n'y a point de divinité hormis Allāh, Muhammad est l'Envoyé de Dieu.

Le nom du défunt était vraisemblablement contenu dans le bandeau de la face opposée. Les deux mots qui restent visibles sur le socle prismatique semblent correspondre à ذكر له souvenir de Dieu, expression absent du Coran ${ }^{14}$, ce qui est intéressant, les socles de ce type portant généralement des versets.

13 Trois de ces tombeaux sont visibles sur le cliché photographique. Nous remercions Martina Massullo de nous avoir fourni l'indication du nom de la ziyāra. Pour des renseignements concernant ce petit cimetière, localisé à l'est de la citadelle en direction des collines de Rawza, voir également Massullo 2017.

14 Dans le Coran nous retrouvons uniquement les expressions ذكر الله (Cor. LVIII, 19) ; دك ذكر الله (Cor. V, 91 ; Cor. XXIV, 37; Cor. LXIII, 9); إذا ذكر الله (Cor. VIII, 2 ; Cor. XXII, 35 ; Cor. XXXIX, 45) ; الله ذكر (Cor. XXXIX, 22) ; إلى ذكر الله (Cor. XXXIX, 23 ; Cor. LXII, 9). 


\section{ELEMENTS ISOLES}

Dans cette section nous prenons en examen les éléments de tombeaux isolés et pourvus d'une partie de l'épitaphe. Il s'agit de neuf couronnements (fig. 12-37) et de quatre socles (fig. 38-42).

\subsection{Les blocs de couronnement}

Tous les blocs dont il est question appartiennent à la même typologie de couronnements monolithiques et moulurés dont le côté latéral affecte la forme d'un arc/fleuron trilobé ${ }^{15}$. Deux spécimens (inv. C5812 et C5817) furent retrouvés en 1958, durant les fouilles du palais ghaznévide, remployés dans une petite nécropole qui, bien après la chute de la dynastie, avait été installée devant la façade de l'édifice.

Inv. C5812. Ce petit fragment $(20,5 \times 20 \times 10,5 \mathrm{~cm})$, exposé au Musée Islamique de Rawza, fut déplacé pendant les années du conflit dans les dépôts du Musée National de Kaboul, où il se trouve encore aujourd'hui ( $\mathrm{n}^{\circ}$ inv. du musée 05.2.1243). Le texte, dont ils ne restent que le début et la fin, était sculpté en écriture cursive - vraisemblablement dépourvue de points diacritiques - sur les deux faces longitudinales du bloc, à l'intérieur de deux bandeaux rectangulaires. L'expression اغفر لـ, (O Dieu) pardonne à, introduisait le nom du défunt (fig. 12). Le dernier mot du texte sculpté à la fin du bandeau de la face opposée étant incomplet, sa lecture nous échappe, mais il devait terminer la séquence des éléments du nom (fig. 13). Le ductus des quelques lettres restantes pourrait reconduire au mot السلالطين], les/des sulțāns, et avoir fait partie d'un laqab. Cependant, cette hypothèse est soulevée avec beaucoup de prudence, un laqab de ce type, à l'époque ghaznévide, ayant appartenu uniquement à un souverain ${ }^{16}$.

Inv. C5817. Le deuxième élément a été également découvert dans une forme fragmentaire $(20 \times 31 \times 8 \mathrm{~cm})$. En 1966 il fut transféré au Musée National d’Art Oriental de Rome $\left(\mathrm{n}^{\circ} \text { inv. du musée } 7782\right)^{17}$. Ce fragment présente cinq portions de bandeaux épigraphiques en écriture cursive pourvue de points diacritiques (fig. 14-16).

Face est, partie centrale :

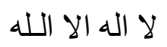

Il n'y a point de divinité hormis Allāh

Face ouest, partie centrale :

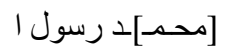

[Muha]mmad est l'Envoyé de

15 Voir Giunta 2003, 333-344.

16 En effet, les trois seules occurrences de ce terme se retrouvent dans les laqabs مولى et ملك السلاطين ,سيد السلاطين السلاطين, qui sont sculptés sur des éléments de décoration architectonique en marbre provenant de la ville. Les deux premiers laqabs rentrent dans le protocole officiel du sulțān Ibrāhīm fils de Mas 'ũd I (1059-1099), le dernier dans celui de son fils, le sulțān Mas'ūd III (1099-1115). Pour ces laqabs voir Giunta - Bresc 2004, 190, 198, 200.

17 Pour des renseignements à propos du déplacement en Italie d'une partie des objets retrouvés par la Mission Archéologique Italienne, voir Giunta 2005. 
Face nord, partie centrale :

$$
\begin{gathered}
\amalg \\
\text { Dieu }
\end{gathered}
$$

Face est, sommet :

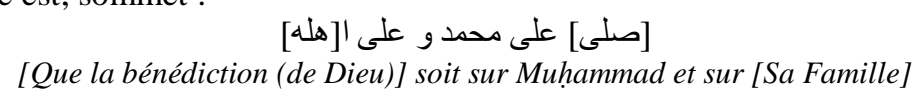

Face ouest, sommet :

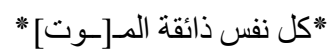

*Tout homme goûtera la mo[rt]* (Cor. XXIX, 57).

Malgré la longueur de cette partie de l'épitaphe, il n’y a aucune indication sur le défunt ${ }^{18}$. La šahāda et le verset coranique sont fréquemment employés dans les épitaphes de la ville. En revanche, la tașliyya trouve ici sa première attestation à l'époque prise en examen.

Trois blocs de couronnement (inv. Sp98, Sp95, Sp96) proviennent de la ziyārat Ȟwāja Awliyā' - dans la zone méridionale de la ville ${ }^{19}$-, enregistrés en 1966. En 2013 ils étaient dans les dépôts de Ghazni, en très bon état de conservation.

Inv. Sp98. Ce bloc $(75,5 \times 22 \times 17 \mathrm{~cm})$ offre une longue épitaphe très soigneusement exécutée sur les quatre faces de l'élément, à l'intérieur de six bandeaux rectangulaires. De plus, deux ou trois mots sont sculptés sur le côté gauche de la moulure de la face ouest. Les quatre bandeaux de la partie centrale renferment une inscription en coufique fleuri caractérisée par la présence de feuilles bilobées et de demi-palmettes qui agrémentent l'extrémité supérieure des lettres ; quatre éléments en forme de vase générant des fleurons sont sculptés à cheval sur les limites gauche et droite des bandeaux. Les deux bandeaux du sommet présentent un texte en écriture cursive pourvue de points diacritiques (fig. 17-20).

Face est, partie centrale :

$$
\text { بسم الله الرحمن الرحيم *شهد الله }
$$

Au nom de Dieu, le Bienfaiteur, le Miséricordieux *Dieu témoigne

Face sud, partie centrale :

Face ouest, partie centrale :

$$
\begin{gathered}
\text { انهe } \\
\text { que }
\end{gathered}
$$

$$
\text { لا اله الا هو و الملائكة وا(و)لو (ا) العلم قائما با }
$$

"Point de divinité hormis Lui" et avec Lui les anges et ceux qui sont doués

Face nord, partie centrale :

$$
\begin{aligned}
& \text { *قســ(Cor) } \\
& \text { d'intelligence* (Cor. III, 18). }
\end{aligned}
$$

18 Le nom du défunt aurait pu être absent, où figurer sur un élément inférieur du tombeau. A cet égard, voir Giunta 2003, n 1, 5, 9, 14, 15, 17, 18, 28, 35, 38.

19 Quatorze épitaphes de tombeaux appartenant à cette ziyāra avaient déjà été lues au tout début du XX $\mathrm{X}^{\mathrm{e}}$ siècle par Muḥammad Riḍā et publiées en 1967 (Riḍā 1967, 92, 182, 209 ; Laviola 2010-2011, 64). Une épitaphe attribuée au XII ${ }^{\mathrm{e}}-\mathrm{XIII}{ }^{\mathrm{e}}$ siècle, retrouvée par la Mission italienne dans cette ziyāra en 1957, figure dans notre étude monographique (Giunta 2003, $\mathrm{n}^{\circ}$ 53) ; toutes les autres épitaphes datent à partir de la deuxième moitié du XVe siècle. A propos des erreurs fréquents dans les lectures fournies par Riḍā, voir Giunta 2003, 10. 
Face est, sommet :

بسم الله [الرحمن ا]لرحيم *كل نفس ذائقة المـوت ثم الينا ترجعون * في يوم الرا[بع]

Au nom de Dieu, [le Bienfaiteur, le] Miséricordieux *Tout homme goûtera la mort.

Vous serez, ensuite, ramenez vers nous* (Cor. XXIX, 57). Dans le jour 4

Face ouest, sommet :

$$
\begin{aligned}
& \text { من شهر الله المباررك شعبا؟]ن سنة خمس و تسعين و خمس ماية [...] } \\
& \text { du mois bé[ni] d'Allāh [ša 'bā ?]n de l'année cinq et quatre-vingt-dix et cinq cents } \\
& \text { [...] (4 ša bān [?] } 595 \text { / } 8 \text { juin [?] 1199). }
\end{aligned}
$$

Les bandeaux sculptés sur la partie centrale des quatre faces du bloc offrent un verset coranique, d'usage fréquent à Ghazni ${ }^{20}$, introduit par la basmala. Une deuxième basmala, suivie du verset coranique XXIX, 57, est exécutée sur le sommet du bloc. Cette partie précède les informations relatives à la date du décès qui reste parfaitement lisible. En revanche, nous n'arrivons pas à proposer une lecture du dernier mot de ce bandeau, ainsi que des quelques mots sculptés en relief sur la moulure. Un déchiffrement de l'épitaphe a également été proposé par Riḍā ${ }^{21}$. Nous avons relevé deux discordances dignes d'attention : l'auteur omet le nom du mois qui, malgré partiellement effacé et dont la lecture est douteuse, suit le mot al-mubārak; pour le chiffre des dizaines il propose سبعين (soixante-dix) au lieu de تسعين (quatre-vingt-dix). La présence des deux points diacritiques au-dessus de la première lettre de ce mot est toutefois certaine.

Inv. Sp95. L’épitaphe de ce bloc $(65,5 \times 24,5 \times 15 \mathrm{~cm})$ est également sculptée sur les faces longitudinales, soit sur la partie centrale, soit sur le sommet. Dans les deux cas, l'écriture est en caractères cursifs, partiellement diacrités et vocalisés. De nombreux petits cercles - qui jouent parfois la fonction de sukūn - se distribuent au-dessus des lettres. Un listel sculpté en relief dessine sur les faces latérales du bloc un motif en forme d'arc trilobé qui reproduit, de façon schématique, le profil latéral du bloc (fig. 21-24).

Face est, partie centrale :

$$
\text { العبد المذنب الى رحمة الله تعالى }
$$

L'esclave pécheur qui a soif de la miséricorde de Dieu, le Très-Haut

Face ouest, partie centrale :

$$
\begin{gathered}
\text { ابو بكر الحسن معروف دهستاني } \\
\text { Abü Bakr al-Hasan dit le villageois }
\end{gathered}
$$

Face est, sommet :

$$
\text { لا اله الا الله محمد رسول الله }
$$

Il n'y a point de divinité hormis Allāh, Muhammad est l'Envoyé de Dieu

Face ouest, sommet :

$$
\text { بتاريخ قرة (sic) ماه رجب سنة تسع و تسعين }
$$

En date du premier jour du mois de rağab de l'année neuf et quatre-vingt-dix.

L'épitaphe de ce bloc contient les renseignements principaux relatifs au défunt. Seul est omis le chiffre des centaines de la date, curieusement fourni par Riḍā qui lit $399^{22}$.

20 Ce verset coranique a été enregistré 21 fois dans les épitaphes déjà publiées (Giunta 2003, 364-365).

21 Riḍā 1967, 183

22 Riḍā 1967, 182-183. Parmi les autres anomalies dans le déchiffrement du texte donné par Riḍā, signalons alHusayn au lieu d'al-Hasan (pourtant, ce mot est clairement vocalisé), ainsi qu'al-ma 'rū $f$ au lieu de ma 'rūf. 
Or, sur la base de nos connaissances, une datation de ce bloc à la fin du IVe siècle de l'hégire (fin $\mathrm{X}^{\mathrm{e}}$ siècle) est à exclure, surtout en vertu de l'emploi du cursif qui n'apparaît que durant la première moitié du siècle suivant ${ }^{23}$. En effet, nous avons le choix entre 499/1106-7 et 599/1203-4, avec une forte présomption en faveur de la première date qui tomberait durant le règne du Ghaznévide Mas 'ūd III ${ }^{24}$. En ce qui concerne la date, notons également que le mot غرة incorrectement transcrit sous la forme de قرة ; persan ماه. ${ }^{25}$.

Inv. Sp96. La distribution de l'épitaphe sur le troisième bloc de la ziyāra $(65,5 \times 26 \times 11$ $\mathrm{cm})$ suit une organisation assez singulière : la face orientale porte trois lignes de texte respectivement sculptées sur le sommet, sur la section centrale et sur la base; la face opposée présente une seule ligne sur la partie centrale. Le style de la graphie cursive, pourvue de points diacritiques, diffère sensiblement d'une ligne à l'autre, ce qui donne l’impression d’un résultat obtenu par plusieurs lapicides (fig. 25, 26).

Face est, partie centrale :

$$
\text { العبد علي يشير بغدادي حاجي المسترشدي }
$$

L'esclave 'Alī Yašîr Bag̀dādì Hāğğğ le Grand Maître

Face ouest, partie centrale :

Face est, sommet :

$$
\begin{aligned}
& \text { بتاريخ الخامس والعشرين من الثهر الله (sic) [...] } \\
& \text { en date du } 25 \text { du mois d'Allāh [...] }
\end{aligned}
$$

$$
\text { Il n'y a point de divinité hormis Allāh, Muhammad est l'Envoyé de Dieu. }
$$

L'épitaphe révèle le nom et la date de mort du défunt qui provenait de la ville de Baghdad. La date comprend uniquement le jour du mois. Les quelques mots sculptés sur la base de la face orientale du bloc sont si embrouillés qu'il est difficile de trouver un significat en les lisant de droite à gauche. Nous avançons l'hypothèse qu'un lapicide pas trop expert ait voulu transcrire une expression fréquemment employée dans l'incipit des épitaphes beaucoup plus tardives إلى حريم العقبى, vers le lieu sacré de l'au-delà éternel, sans toutefois respecter la disposition correcte des trois mots ${ }^{26}$.

Deux blocs de couronnement, enregistrés en 1966 dans un espace funéraire localisé à l'est du mausolée de Muhammad Šarīf Hāān ${ }^{27}$, ont été retrouvés dans les dépôts de Ghazni en 2004 (inv. Sp90 et Sp92).

23 Giunta 2001.

24 Cette hypothèse se base soit sur le style de la graphie cursive, soit sur le décor des faces latérales du bloc.

25 La question relative à la présence de mots en persan dans les épitaphes en arabe de la ville a été abordée dans Giunta 2010 et Allegranzi 2015.

26 Pour les attestations de cette expression dans sa forme complète (قد ارتحل من دار الدنيا الى حريم العقبى, il s’est transporté de la demeure terrestre périssable vers le lieu sacré de l'au-delà éternel), voir les épitaphes datables du XVII'-XVIII ${ }^{\mathrm{e}}$ siècles publiées dans Riḍā 1967, 185-191, 196-202, 204, 209-218 ; voir également Massullo 2017, $\mathrm{n}^{\circ}$ 6, 25, 35, 49, 50, 71, 80, 85, 86, 92, 102, 112, 113, 117, 134.

27 A propos de ce petit cimetière et du mausolée qui porte ce nom, voir Massullo 2017 (catalogue, section VI). Pour un autre tombeau du XII ${ }^{\mathrm{e}}$-XIII ${ }^{\mathrm{e}}$ siècle provenant de ce même espace funéraire voir Giunta 2003, n 54. 
Inv. Sp90. Le bloc est en bon état de conservation. L’épitaphe se déroule dans les deux bandeaux de la section centrale des faces longitudinales et s'achève sur le sommet de la face orientale. Dans le premier cas, il s'agit d'une écriture cursive non-diacritée qui se détache sur un fond nu ; la partie du texte sculptée sur le sommet est dépourvue de cadre et elle est exécutée en écriture coufique (fig. 27, 28).

Face est, partie centrale :

$$
\text { بسم الله الرحمن الرحيم *شهد الله ا }
$$

Au nom de Dieu, le Bienfaiteur, le Miséricordieux *Dieu témoigne

Face ouest, partie centrale :

$$
\text { *نه لا اله الا هو و الملائكة وا(ولوا) : نان }
$$

“Point de divinité hormis Lui” et avec Lui les anges et les po(ssesseurs)*(Cor. III, 18)

Face est, sommet :

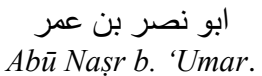

Remarquons ici une inversion dans l'emploi du cursif et du coufique ; en effet, contrairement à un usage courant dans la ville, la partie «historique » du texte est transcrite en coufique ${ }^{28}$.

Inv. Sp92. Le deuxième bloc présente un texte totalement en cursif non-diacrité et dépourvu de cadre, qui se déroule sur les quatre faces et s'achève sur le sommet de la face orientale (fig. 29-32).

Face nord, partie centrale :

$$
\text { هذه }
$$

Face est, partie centrale :

$$
\text { روضة القاضي الامام اجل بها }
$$

tombe appartient à al-Qāḍ̄ al-Imām ăgall Bahā

Face sud, partie centrale :

$$
\text { الدين }
$$

Face ouest, partie centrale :

$$
\text { ابو المعالي بن محمد بن احمد الصغاني رحمه الله }
$$

Abū al-Ma àlī b. Muhammad b. Ahmmad al-Ṣag̀ānī. Que Dieu ait pitié de lui !

Face est, sommet :

$$
\begin{gathered}
\text { في شهور سنة ثمان و ثمانين } \\
\text { dans l'un des mois de l'année huit et quatre-vingt. }
\end{gathered}
$$

Encore une fois l'épitaphe du bloc dévoile le nom du défunt et la date de sa mort mais la date est à nouveau dépourvue du chiffre de l'année, malgré l'espace à la disposition du lapicide. L'incipit du texte contient la désignation de la tombe pour

28 Voir Giunta 2003, 431-432. 
laquelle le mot روضة, jardin paradisiaque, est utilisé ${ }^{29}$. La nisba du défunt est attestée dans d'autres épitaphes de la ville, dont l'une datée de 506/1113-4 ${ }^{30}$.

En 1966, à l'intérieur du mausolée de Muhammad Šarīf Ḩān, à côté de sept tombeaux datés à une période comprise entre 1544 et $1611^{31}$, se trouvait également un petit fragment de couronnement (inv. IG6202) dont la forme, le type d'épitaphe et le style de l'écriture trahissent une réalisation à une époque plus ancienne (fig. 33). Le cliché photographique reproduit uniquement la face orientale :

$$
\begin{aligned}
& \text { [بسـ] الله الرحمـ[ن] } \\
& \text { [Au no]m de Dieu, le Bienfaite[ur]. }
\end{aligned}
$$

Un autre bloc de couronnement cassé en deux parties qui s’ajustaient parfaitement (inv. IG6203) a été retrouvé dans le masğid Haḍrat-i Šams en 1962. Il était placé sur le côté d'un tombeau plus tardif ${ }^{32}$, appuyé contre l'une des parois de l'édifice. La photo reproduit la partie de l'épitaphe en cursif de l'une des faces longitudinales, vraisemblablement la face ouest de la tombe (fig. 34).

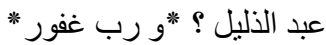

$$
\begin{aligned}
& \text { 'Abd al-dalīl ? *un Seigneur qui pardonne* (Cor. XXXIV, 15). }
\end{aligned}
$$

Les cinq mots qui restent visibles sur ce bloc contiennent la partie finale du nom du défunt suivie de la partie finale d'un verset coranique. Le nom de 'Abd al-Dalīl se retrouve dans une autre épitaphe de la ville dédiée à un personnage provenant de la ville de Balkh ${ }^{33}$.

Nous ignorons la provenance des trois derniers blocs de couronnements inédits (inv. IG1180, IG1181, IG1143) qui, en 2013, étaient conservés dans les dépôts de Ghazni. Par ailleurs, les clichés photographiques reproduisent uniquement l'une des faces longitudinales de chaque bloc.

Inv. IG1180. Le bloc est pourvu d'un bandeau rectangulaire renfermant une inscription cursive. Des éléments végétaux typiques du répertoire ornemental ghaznévide comblent les espaces vides entre les lettres. Nous n'arrivons pas à proposer une lecture du texte qui, par ailleurs, pourrait avoir été transcrit en langue persane (fig. 35).

Inv. IG1181. Le bandeau épigraphique sculpté sur ce bloc occupe toute la longueur de la face visible sur la photo. L’écriture cursive est pourvue de points diacritiques (fig. 36).

$$
\text { * كل من عليها فان و يبقى وجه ربك ذو الجلال و الاكران " }
$$

*Tout ce qui se trouve sur la terre disparaîtra. La face de ton Seigneur subsiste, pleine de majesté et de munificence* (Cor. LV, 26-27).

Inv. IG1143. A l’instar du spécimen précèdent un long bandeau rectangulaire se déroule sur la section centrale du bloc. L’écriture cursive n’est pas diacritée (fig. 37).

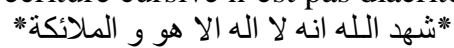

*Dieu témoigne "Point de divinité hormis Lui" et avec Lui les anges* (Cor. III, 18).

\footnotetext{
Pour d'autres attestations, voir Giunta 2003, n 54

Riḍā 1967, 157-161.

Massullo 2017, n 29-35.

Massullo 2017, n IG6027.

Rị̣ā 1967, 191.
} 


\subsection{Les socles}

Parmi les autres éléments de tombe pourvus d'inscriptions et inédits signalons, pour conclure, quatre fragments de socles (inv. C2897, Sp74, IG319, IG496). Exception faite pour le premier spécimen qui est à degrés, tous ces socles affectent la forme d'un prisme à section trapézoïdale.

Inv. C2897. Ce fragment, divisé en deux morceaux $(28 \times 25 \times 9 \mathrm{~cm})$ qui, vraisemblablement, n'étaient pas directement agencés, a été récupéré par la Mission en 1957 à l'intérieur de la ziyāra dite d'Ibrāhīm, construite aux XV-XVI siècle à l'intérieur du périmètre de l'’̄wān ouest du palais royal ghaznévide ${ }^{34}$. En 2004, il a été retrouvé dans le même état de conservation dans les dépôts du Musée National de Kaboul (inv. du musée 05.2.1571 ; fig. 38). L'inscription, en coufique à peine fleuri, est sculptée en relief sur le bord du degré supérieur.

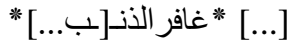

$$
\begin{aligned}
& \text { [...] *Celui qui pardonne le péché* [...] (Cor. XL, 3). }
\end{aligned}
$$

Nous arrivons à proposer uniquement le déchiffrement de la partie de texte du morceau de gauche qui contient les deux premiers mots d'un verset coranique.

Inv. Sp74. Ce fragment $(41 \times 26 \times 6,5 \mathrm{~cm})$ formait l'un des angles d'un socle prismatique retrouvé en 1966 dans un petit cimetière localisé au nord du minaret de Bahrām Šāh. Il est actuellement conservé dans les dépôts du Musée National d’Art Oriental de Rome ( $\mathrm{n}^{\circ}$ inv. du musée 7813). L'inscription est exécutée en coufique simple; les terminaisons supérieures des corps des lettres sont agrémentées de pointes effilées (fig. 39).

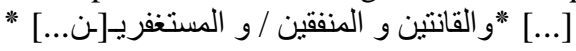

* [... pour ceux qui sont patients, sincères] et pieux, pour ceux qui font l'aumône / et qui implorent le pardon de Dieu [...]* (Cor. III, 17).

Inv. IG319. Ce deuxième fragment d'angle de socle, aujourd'hui disparu, a été pris en photo en 1977, lorsqu'il était remployé sur la paroi extérieure d'une maison moderne de la ville. L'inscription est sculptée en cursif dépourvu de points diacritiques (fig. 40, 41).

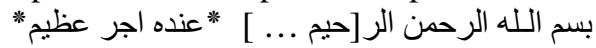

Au nom de Dieu, le Bienfaiteur, le Mi[séricordieux...] * une récompense sans limites se trouve auprès de Dieu* (Cor. VIII, 28).

Nous supposons que le socle contenait uniquement la basmala, suivie du verset coranique VIII, 28 dont ils ne restent que les trois derniers mots.

Inv. IG496. Ce troisième angle d'un socle prismatique $(28 \times 20 \mathrm{~cm})$ est absent de l'ancienne documentation acquise par la Mission ; en effet, il a été retrouvé en 2004 dans les dépôts du Musée National de Kaboul (sans $n^{\circ}$ inv.). Une inscription en cursif diacrité est sculptée sur le rebord de l'élément (fig. 42).

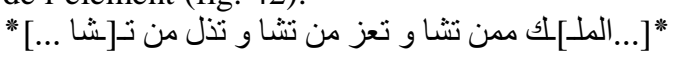

[*...Tu enlèves la royau]té à qui Tu veux. Tu honores qui Tu veux et Tu abaisses qui Tu v[eux...*] (Cor. III, 26).

\footnotetext{
34 En ce qui concerne les éléments en marbre remployés dans cette ziyāra, voir Laviola 2010-2011, 46.
} 
Les tombeaux de ce petit recueil forment un groupe assez homogène et font partie de la plus ancienne production funéraire d'époque islamique de la ville. Sur la base de nos connaissances, ainsi que des comparaisons avec les tombeaux publiés en 2003, certains spécimens remontent à l'époque ghaznévide, tels les trois tombeaux fouillés aux alentours de la ziyāra de Šams (fig. 3-10) ; les deux fragments de blocs de couronnement fouillés à l'entrée du palais ghaznévide (fig. 12-16) ; les blocs de couronnement inv. Sp95 (fig. 2124), Sp90 (fig. 27-28), IG6202 (fig. 33), IG1180 (fig. 35), IG1181 (fig. 36), IG1143 (fig. 37); les socles inv. C2897 (fig. 38) et Sp74 (fig. 39) ${ }^{35}$. L'un des spécimens provenant de la ziyārat Howāja Awliyā' (Sp98, fig. 17-20) est daté de 595/119936. Pour tous les autres tombeaux (fig. 1, 2, 11, 25, 26, 29-32, 34, 41, 42), une datation entre la fin du XII ${ }^{\mathrm{e}}$ siècle et le XIII ${ }^{\mathrm{e}}$ siècle peut être proposer avec beaucoup de probabilité.

\title{
BIBLIOGRAPHIE
}

\author{
AllegranZI, V. \\ 2015 The Use of Persian in Monumental Epigraphy from Ghazni: Eurasian Studies 13 (2015), \\ BOMBACI, A \\ p. 23-41. \\ 1966 The Küfic Inscription in Persian Verses in the Court of the Royal Palace of Mas 'üd III at \\ GIUNTA, R. \\ Ghazni, Rome 1966. \\ 1999 Les inscriptions de la ville de Ġaznī (Afghanistan), 3 vol. (Thèse de doctorat, Université \\ de Provence “Aix-Marseille I”, 1999). \\ 2000 Ghaznī. ii. Monuments and Inscriptions: Encyclopaedia Iranica X/4 (1989), p. 385-388. \\ 2001 The Tomb of Muhammad al-Harawī (447/1055) at Gaznī (Afghanistan) and Some New \\ Observations on the Tomb of Mạmūd the Gaznavid: East and West 51/1-2 (2001), p. \\ 109-126. \\ 2003 Les inscriptions funéraires de $\dot{G} a z n \bar{\imath}$ (IVe-IXe/Xe-XVe siècles) (Università degli Studi di \\ Napoli “L’Orientale”, Dipartimento di Studi Asiatici Series Maior VIII - IsIAO, Roma - \\ Fondation Max van Berchem, Genève), Naples 2003. \\ 2005 Islamic Ghazni. An IsIAO Archaeological Project in Afghanistan. A Preliminary Report \\ (July 2004-June 2005): East and West 55 (2005), p. 473-484. \\ 2010 Les inscriptions persanes dans l'épigraphie monumentale de la ville de Ghazni \\ (Afghanistan) aux 6e-7e/12e-13e siècle: C.G. CERETI (ed.), Iranian Identity in the Course \\ of History: Proceedings of the conference Held in Rome, 21-24 September 2005 (IsIAO. \\ Serie Orientale Roma, CV, Orientalia Romana, IX), Rome 2010, p. 163-180.
}

35 Un tombeau ghaznévide inédit et en parfait état de conservation a été exclu de cette étude et il fait l'objet d'une publication en cours. La datation du monument pouvant s'inscrire dans les premières années de règne de la dynastie, l'analyse de son aspect morphologique, du contenu des inscriptions et des styles de l'écriture demande d'être conduite en profondeur.

36 Le type de coufique représente une évolution de celui qui avait été utilisé dans l’inscription en langue persane des plaques en marbre du palais royal de la ville (Bombaci 1966). 
GiUNTA, R. - BRESC, C.

2004 Listes de la titulature des Ghaznavides et des Ghurides à travers les documents numismatiques et épigraphiques : Eurasian Studies III/2 (2004), p. 161-243.

LAVIOLA, V.

2010-2011 Le ziyarat di Ghazni nell'opera di Riḍa e negli archivi della missione archeologica italiana in Afghanistan (Mémoir de master, Université de Naples “L’Orientale”, 2010-11 ; inédit).

Massullo, $\mathrm{M}$

2017 Les tombeaux et les épitaphes de Ghazni (Afghanistan) entre XVe-XVIII ${ }^{e}$ siècles (Thèse de doctorat, Université Aix-Marseille - Université de Naples “L’Orientale”, 2017 ; inédit).

RIḌA, M.

1967 Riyāẹ al-alwāh muštamil bar katībehā-ye qubūr wa abniya-ye Ġazna (deuxième frontispice Riyaz ul-alvah. Inscriptions from Ghazna), Kabul 1967. 


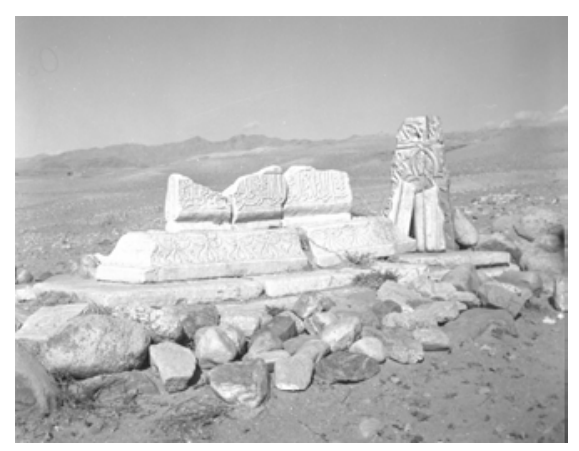

Fig. 1 - Tombeau dans un cimetière inconnu, inv. IG485 (C IsIAO, Neg. R 5315/18).

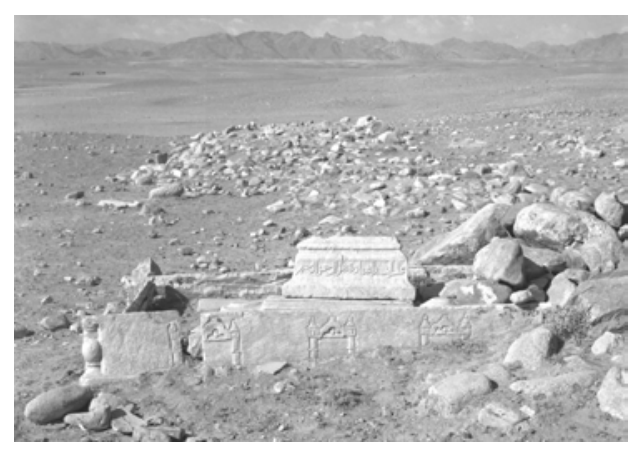

Fig. 2 - Tombeau dans un cimetière inconnu, inv. IG491 (C) IsIAO, Neg. R 5315/19).

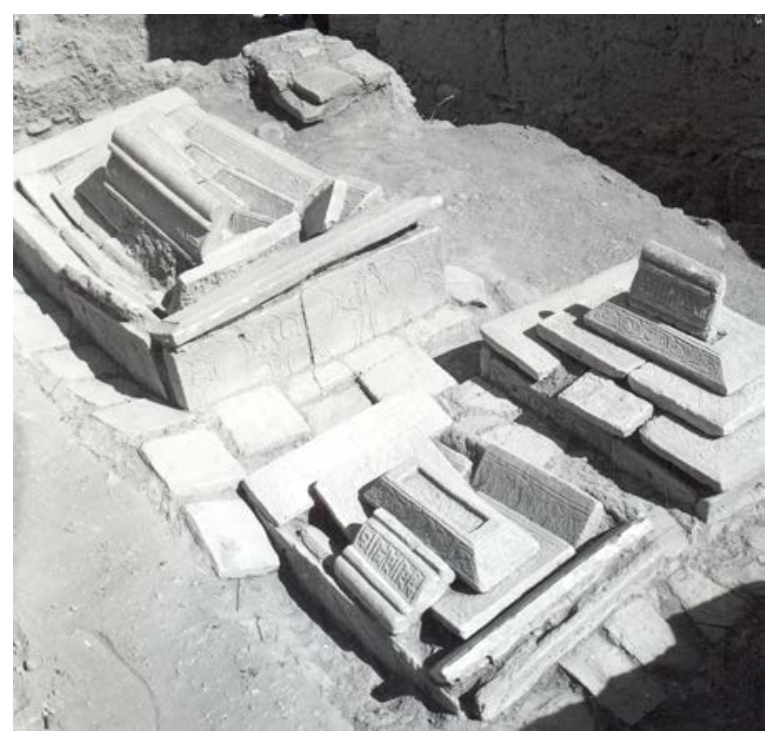

Fig. 3 - Les trois tombeaux fouillés à proximité de la ziyāra de Šams : inv. IG1811 en haut ; inv. IG57 en bas à gauche ; inv. IG6200 en bas à droite (C IsIAO, Neg. R 8153/12).

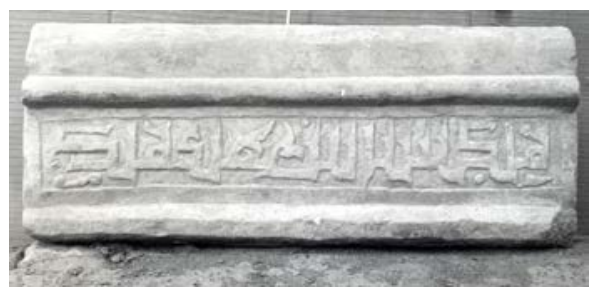

Face est

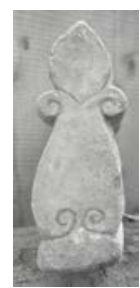

Face sud

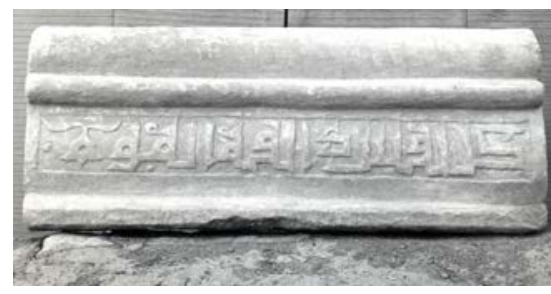

Face ouest

Fig. 4-6 - Couronnement du tombeau inv. IG1811 ( IsIAO, Neg. R 8152/12-14). 


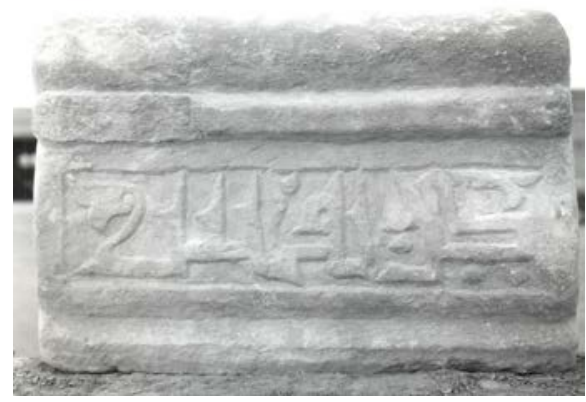

Face est

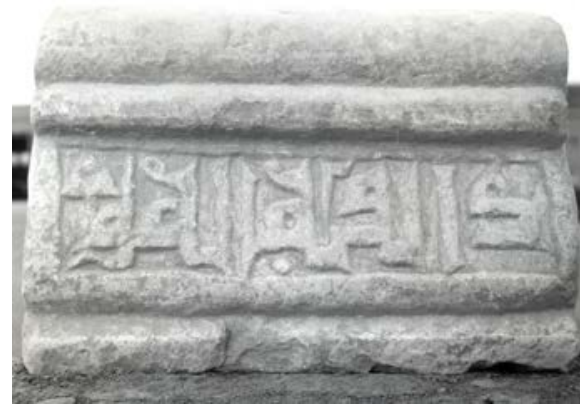

Face ouest

Fig. 7-8 - Couronnement du tombeau inv. IG57 (@ IsIAO, Neg. R 8152/9, $10)$.

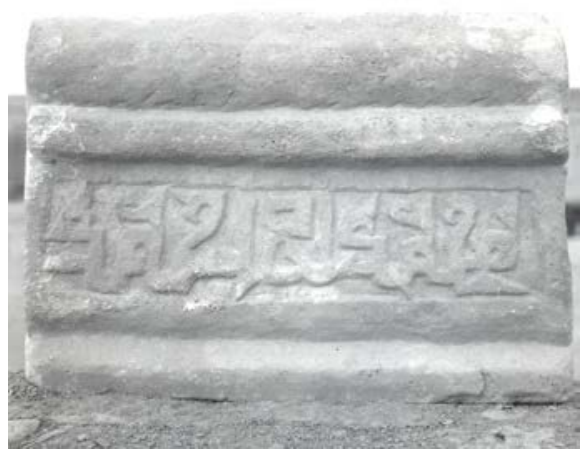

Face est

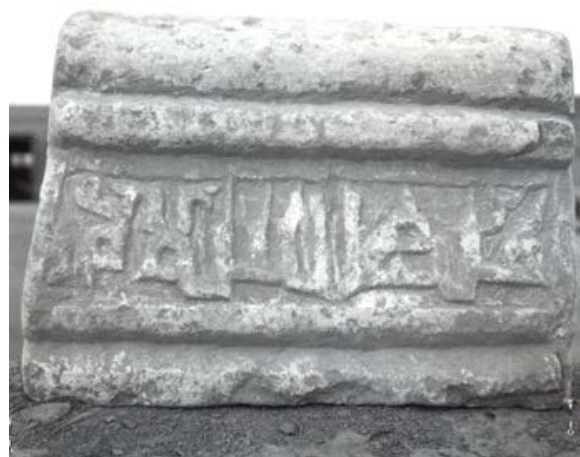

Face ouest

Fig. 9-10 - Couronnement du tombeau inv. IG6200 ( IsIAO, Neg. R 8179/1, 2).

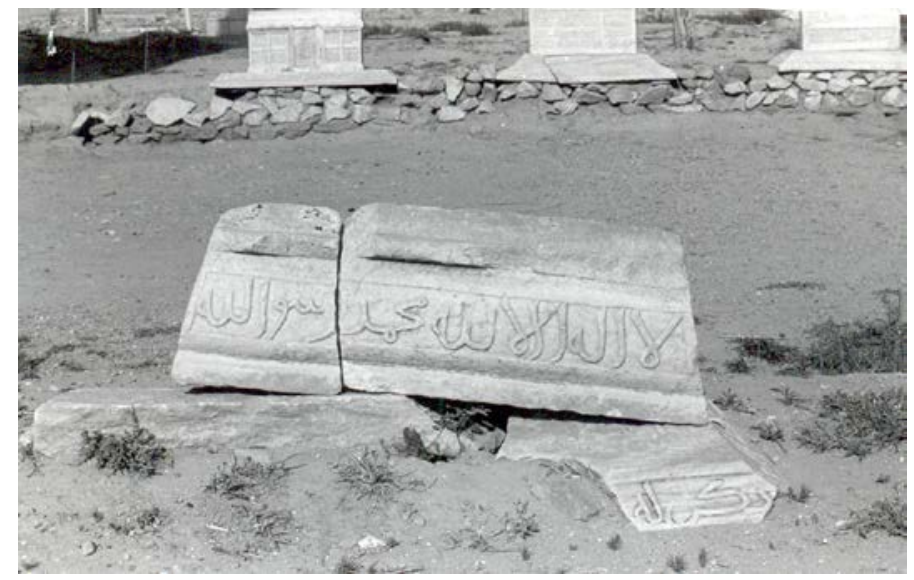

Fig. 11 - Tombeau de la ziyārat 'Alī Lālā, inv. IG6201 (C IsIAO, Neg. 13297/31). 

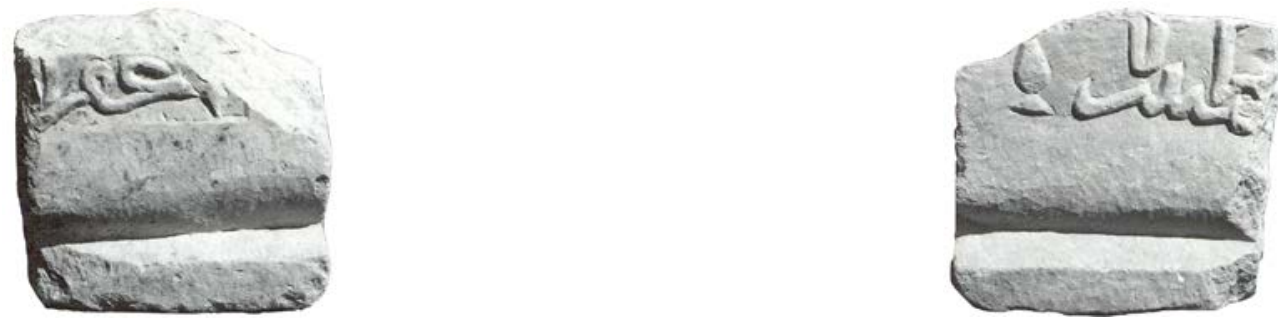

Face est

Face ouest

Fig. 12-13 - Fragment de bloc de couronnement inv. C5812 (C IsIAO, Neg. R 968/2-3).

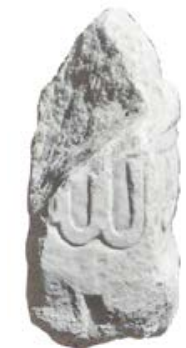

Face nord

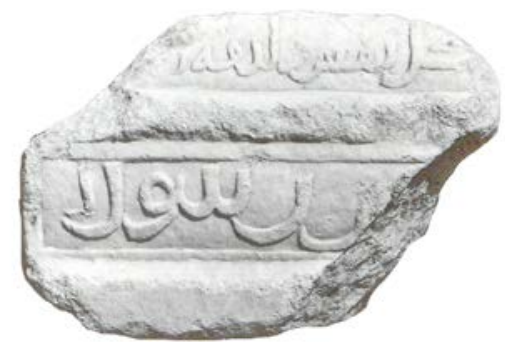

Face ouest

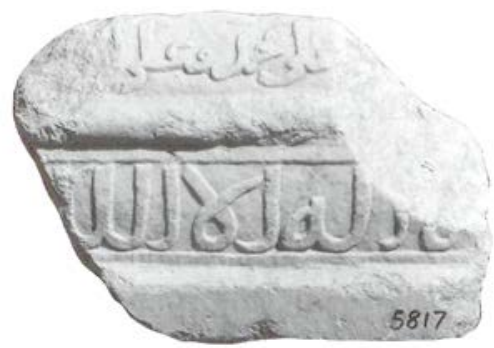

Face est

Fig. 14-16 - Fragment de bloc de couronnement inv. C5817 (C IsIAO, Neg. R 988/7, 9-10).

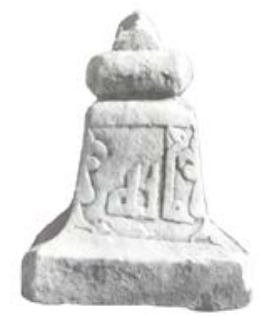

Face sud

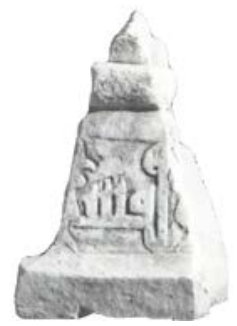

Face nord

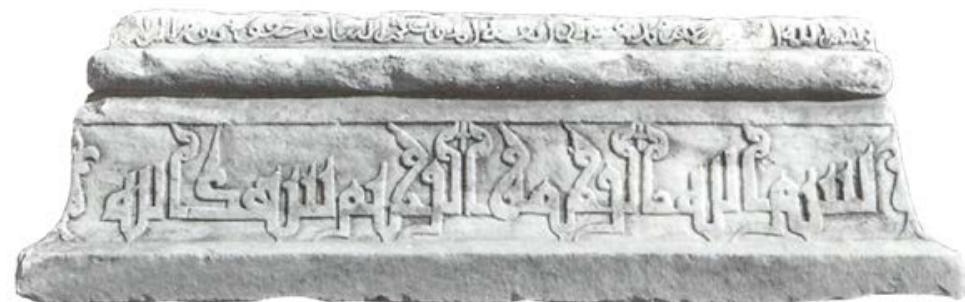

Face est

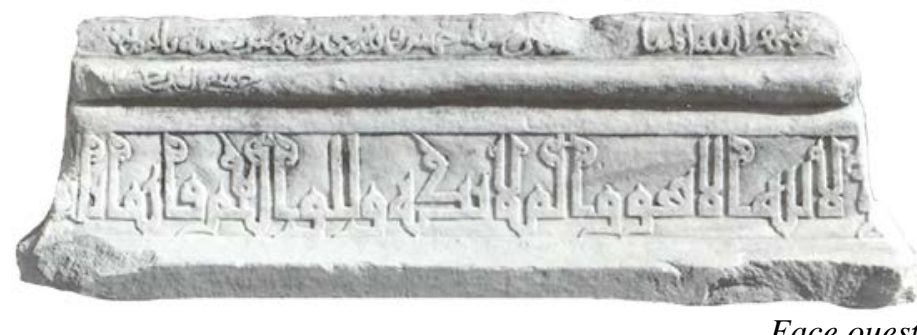

Face ouest

Fig. 17-20 - Les quatre faces du bloc de couronnement inv. Sp98 (@ IsIAO, Neg. R 995/2, $8,1002 / 10,12)$. 


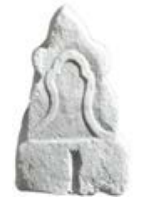

Face nord

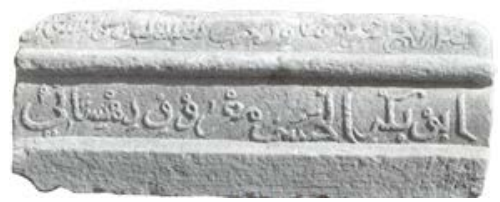

Face ouest

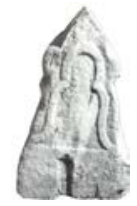

Face sud

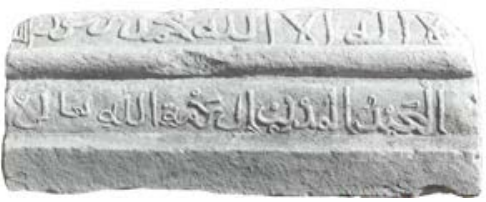

Face est

Fig. 21-24 - Les quatre faces du bloc de couronnement inv. Sp95 (C) IsIAO, Neg. R 1006/5$7,9)$.

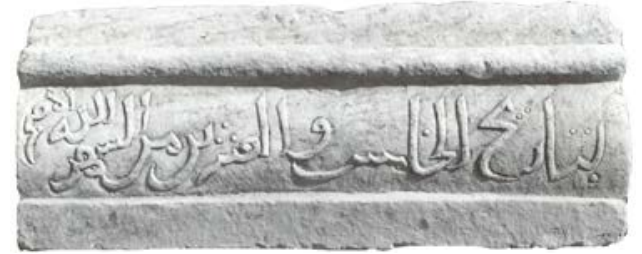

Face ouest

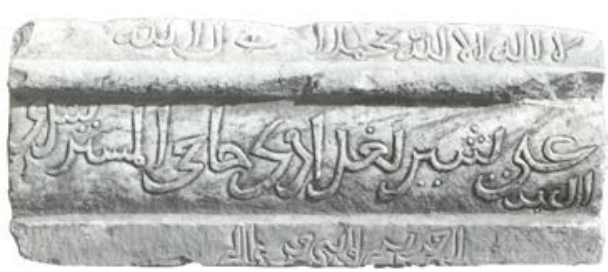

Face est

Fig. 25-26 - Les deux faces du bloc de couronnement inv. Sp96 (ㄷ IsIAO, Neg. R 1006/2$3)$.

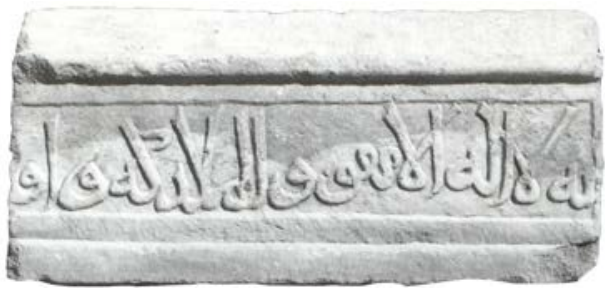

Face ouest

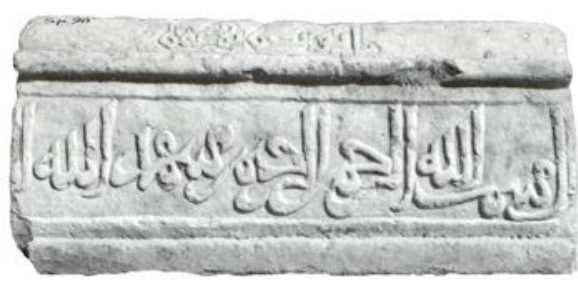

Face est

Fig. 27-28 - Les deux faces du bloc de couronnement inv. Sp90 (ㄷ IsIAO, Neg. R 1002/78). 


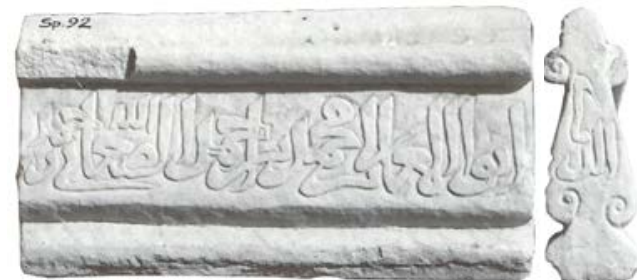

Face ouest
Face sud

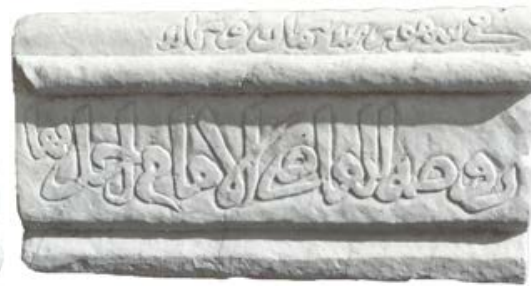

Face est

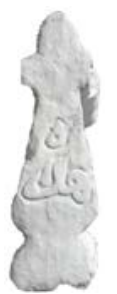

Face nord

Fig. 29-32 - Les quatre faces du bloc de couronnement inv. Sp92 ( IsIAO, Neg. R 996/2, 1002/3-4, 6).

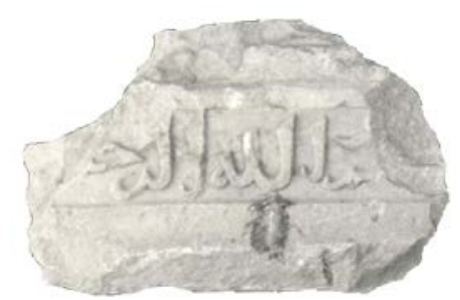

Fig. 33 - Fragment de bloc de couronnement inv. IG6202 (C) IsIAO, Neg. 11017/19).

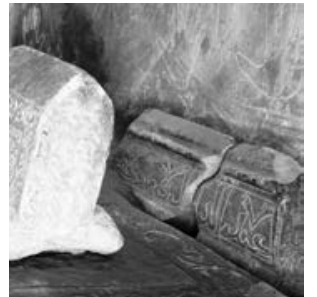

Fig. 34 - Bloc de couronnement inv. IG6203 (C) IsIAO, Neg. R 2229/1).

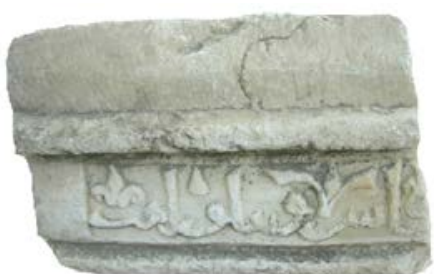

Fig. 35 - Fragment de bloc de couronnement inv. IG1180 (C) IsIAO 2013).

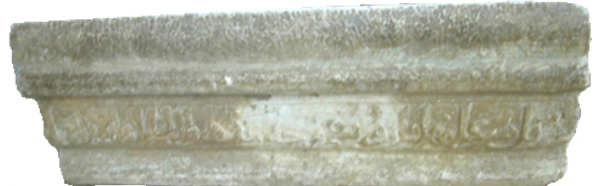

Fig. 36 - Bloc de couronnement inv. IG1181 (C IsIAO 2013).

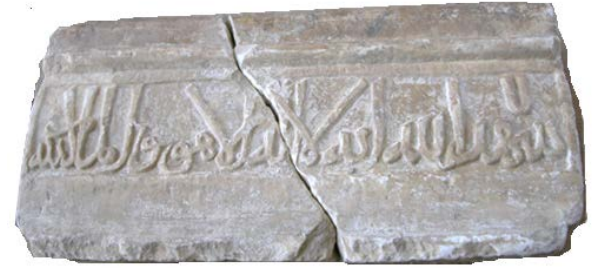

Fig. 37 - Bloc de couronnement inv. IG1143 (C IsIAO 2013). 


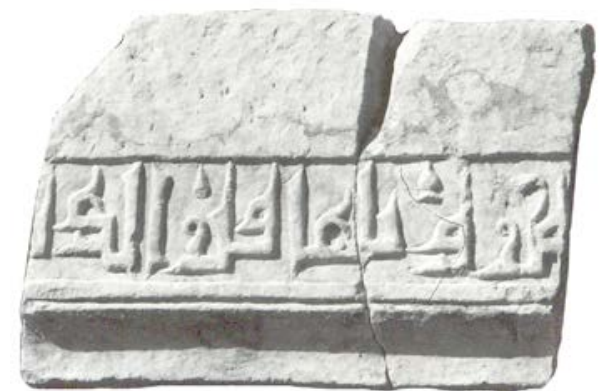

Fig. 38 - Fragment de socle inv. C2897 (C) IsIAO Neg. R 968/4).

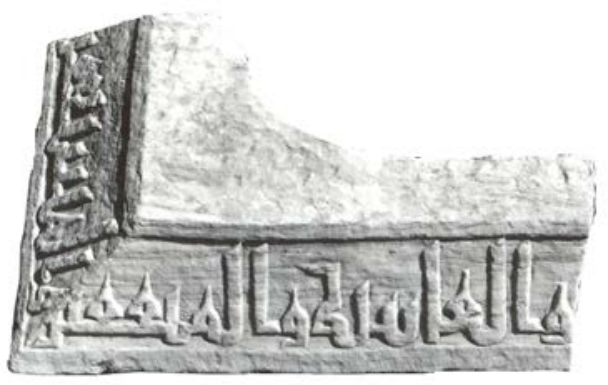

Fig. 39 - Fragment de socle inv. Sp74 (C) IsIAO Neg. R 626/10).

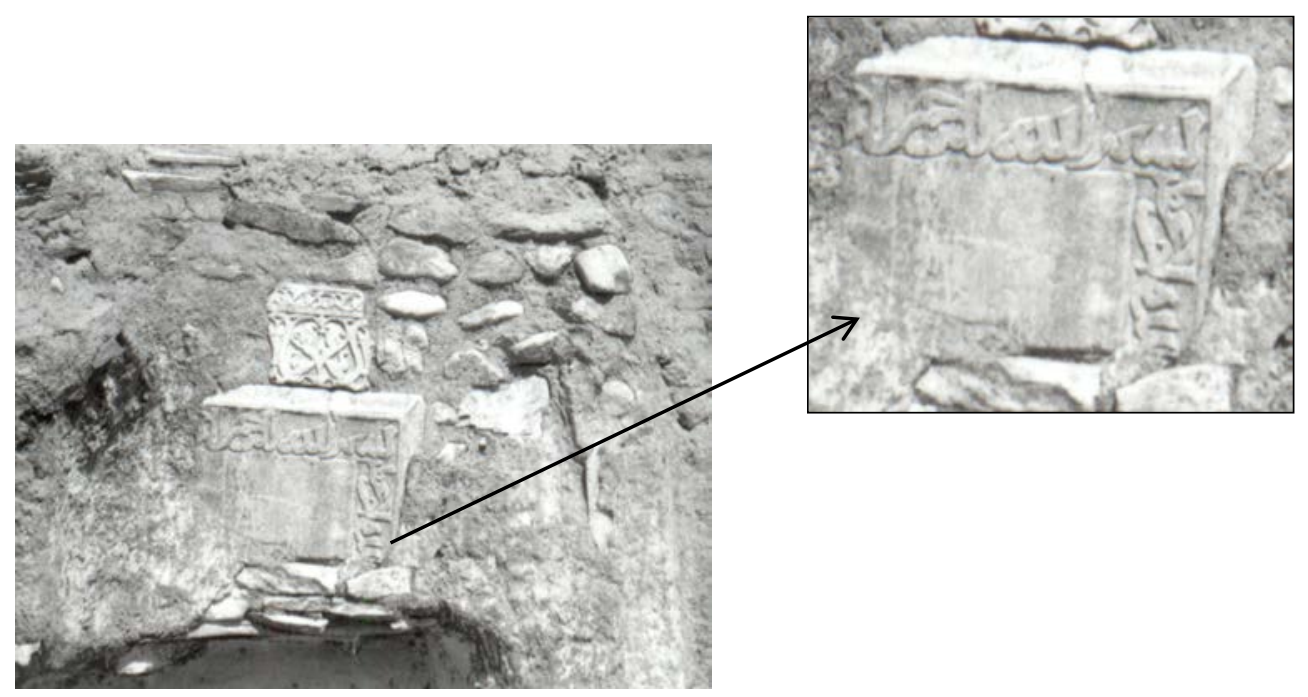

Fig. 40-41 - Fragment de socle de tombe encastré dans la paroi d'une maison moderne (inv. IG319 ; (C) IsIAO Neg. R 12934/8).

Fig. 42 - Fragment de socle inv. IG496 (@) IsIAO 2004).

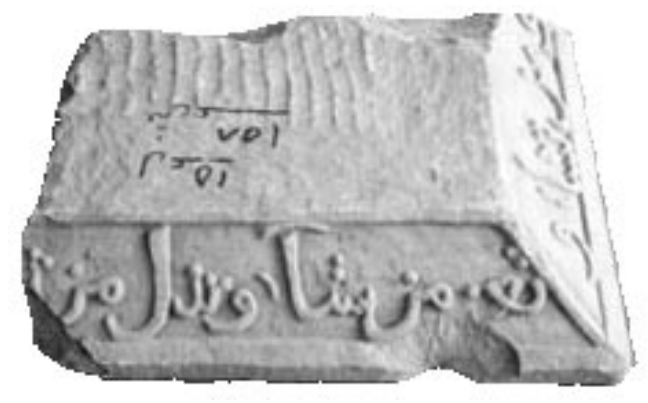

